\title{
Antitumor effect of YM155, a novel small-molecule survivin suppressant, via mitochondrial apoptosis in human MFH/UPS
}

\author{
MASAYA MINODA ${ }^{1}$, TERUYA KAWAMOTO ${ }^{1}$, TAKESHI UEHA ${ }^{2}$, ETSUKO KAMATA ${ }^{1}$, \\ MASAYUKI MORISHITA ${ }^{1}$, RISA HARADA ${ }^{1}$, MITSUNORI TODA ${ }^{1}$, YASUO ONISHI ${ }^{1}$, \\ HITOMI HARA $^{1}$, MASAHIRO KUROSAKA ${ }^{1}$ and TOSHIHIRO AKISUE ${ }^{1,3}$ \\ ${ }^{1}$ Department of Orthopaedic Surgery and ${ }^{2}$ Division of Rehabilitation Medicine, Kobe University \\ Graduate School of Medicine, Chuo-ku, Kobe 650-0017; ${ }^{3}$ Department of Rehabilitation Science, \\ Kobe University Graduate School of Health Sciences, Suma-ku, Kobe 654-0142, Japan
}

Received May 13, 2015; Accepted June 22, 2015

DOI: 10.3892/ijo.2015.3077

\begin{abstract}
Survivin is a member of the inhibitor of apoptosis family, which is known to inhibit mitochondrial apoptosis. Survivin is highly expressed in cancers and plays an important role in cancer cell survival, and increased survivin expression is an unfavorable prognostic marker in cancer patients. YM155, a novel small-molecule survivin suppressant, selectively suppresses survivin expression, resulting in the induction of apoptosis in various malignancies. However, the roles of survivin in human malignant fibrous histiocytoma/ undifferentiated pleomorphic sarcoma (MFH/UPS) have not been studied. In the present study, we examined survivin expression in human musculoskeletal tumor tissues, and the effect of survivin inhibition by siRNA or YM155 on apoptotic activity in human MFH/UPS cell lines. In tumor tissues, mRNA expression of survivin was significantly higher in MFH/UPS samples than in benign schwannomas. Moreover, in vitro studies revealed that both survivin siRNA and YM155 suppressed survivin expression and inhibited MFH/UPS cell proliferation in a dose- and a time-dependent manner. Further, the numbers of apoptotic cells significantly increased with YM155 treatment. In vivo, tumor volume in YM155-treated groups was significantly reduced without significant bodyweight loss. Increased apoptotic activity along with decreased survivin expression was also observed in YM155-treated tumors. The findings in this study strongly suggest that survivin suppressants, including YM155, contribute to the suppression of human MFH/UPS cell growth via promoting mitochondrial apoptosis, and that survivin may be a potent therapeutic target for the novel treatment of human MFH/UPS.
\end{abstract}

Correspondence to: Dr Teruya Kawamoto, Department of Orthopaedic Surgery, Kobe University Graduate School of Medicine, 7-5-1 Kusunoki-cho, Chuo-ku, Kobe 650-0017, Japan

E-mail: trykwmt@med.kobe-u.ac.jp

Key words: survivin, apoptosis, YM155, malignant fibrous histiocytoma/undifferentiated pleomorphic sarcoma, sarcoma

\section{Introduction}

Musculoskeletal malignancies, particularly high-grade sarcomas such as malignant fibrous histiocytoma (MFH), which has recently been classified as undifferentiated pleomorphic sarcoma (UPS), are clinically aggressive and demonstrate high metastatic behavior $(1,2)$. Recent advances have led to multidisciplinary treatments for musculoskeletal malignancies, including surgery, chemotherapy, and radiation therapy, resulting in great improvement in the quality of life of affected patients. Although many chemotherapeutic protocols are used to treat human sarcomas, current therapeutic strategies for high-grade sarcomas, except Ewing's sarcomas and the peripheral primitive neuroectodermal tumors (PNET) family, are ineffective, and result in poor patient prognosis due to local recurrence and metastases (3). Therefore, there is a great need to understand the mechanisms of tumor progression, and new therapeutic strategies against high-grade sarcomas are required.

Survivin is a member of the inhibitor of apoptosis (IAP) family, which is known to inhibit mitochondrial apoptosis. It is expressed in a wide range of embryonic and fetal tissues, but is undetectable in terminally differentiated normal adult tissues, and is highly expressed in most solid and hematological malignancies (4-6). Survivin has been implicated in both cell survival and the regulation of mitosis in tumor cells, and has been consistently identified by molecular profiling analyses to be associated with more advanced disease, abbreviated survival, accelerated rates of recurrence, and resistance to chemotherapy and radiation therapy (7-10). Moreover, elevated survivin expression is an unfavorable prognostic marker correlating with decreased overall survival in a variety of malignant tumors (11). However, there have been few studies that examine survivin expression in musculoskeletal malignancies (12-16).

YM155 is a novel small-molecule that selectively suppresses survivin gene expression, resulting in activation of caspases and induction of apoptosis in malignant tumors (17). The molecular mechanisms underlying YM155-mediated survivin suppression are under evaluation through identification of YM155-interacting molecules that bind to promoter 
regions of the survivin gene (4). Recently, clinical studies have investigated the effects of using YM155 to selectively target survivin on certain cancers (18-20). However, studies have not focused on the effects of survivin suppressants on musculoskeletal malignancies like MFH/UPS.

Based on previous studies, we hypothesized that survivin is highly expressed in human MFH/UPS, and that survivin contributes to tumor progression by inhibiting mitochondrial apoptosis in human MFH/UPS. In the present study, we examined survivin expression in human MFH/UPS tumor tissues and evaluated the effect of survivin inhibition on cell apoptosis using human MFH/UPS cell lines in vitro. For in vivo studies, we used YM155 to characterize the preclinical efficacy profile of survivin.

\section{Materials and methods}

Musculoskeletal tumor tissue samples and human MFH/UPS cell lines. We used 30 human musculoskeletal tumor tissue samples including 15 benign schwannomas (as control) and $15 \mathrm{MFH} / \mathrm{UPS}$ samples. This study was approved by the Kobe University Hospital ethics committee (permission no. 1077), and all patients provided informed consent prior to surgery. The samples were obtained by surgery at Kobe University Hospital in accordance with institutional guidelines and immediately stored at $-80^{\circ} \mathrm{C}$ until use.

Three human MFH/UPS cell lines (Nara-H, Nara-F and TNMY1) were studied in vitro and in vivo. Nara-H and Nara-F were obtained from ScienStuff Co. (Nara, Japan) (21), and TNMY1 was previously established in our laboratory (22). Cells were grown in Dulbecco's modified Eagle's medium (DMEM; Sigma-Aldrich Co., St. Louis, MO, USA) supplemented with $10 \%$ (v/v) fetal bovine serum (Sigma-Aldrich) and $100 \mathrm{U} / \mathrm{ml}$ penicillin/streptomycin solution (Sigma-Aldrich). Cell lines were routinely maintained at $37^{\circ} \mathrm{C}$ in a humidified $5 \% \mathrm{CO}_{2}$ atmosphere. For all experiments, we used DMEM containing $10 \%$ FBS without the antibiotic solution.

siRNA knockdown of survivin in human MFH/UPS cells. To assess the effects of survivin inhibition on apoptotic activity and cell proliferation in human MFH/UPS cells in vitro, we performed siRNA transfection with either survivin-siRNA (Ambion Inc., Austin, TX, USA) or non-specific control siRNA (Ambion Inc.) in MFH/UPS cell lines using Lipofectamine ${ }^{\mathrm{TM}}$ 2000 Transfection reagent, according to the manufacturer's instructions (Invitrogen, Carlsbad, CA, USA). Efficacy of survivin knockdown was assessed by quantitative real-time PCR (qPCR).

Survivin suppressant, YM155. YM155 was purchased from AdooQ BioScience (Irvine, CA, USA), dissolved in dimethyl sulfoxide, and immediately stored at $-80^{\circ} \mathrm{C}$. This stock solution was diluted into culture medium and saline for in vitro and in vivo experiments, respectively, immediately before use.

Human MFH/UPS xenograft studies. Male BALB/c nude mice, aged 5 weeks, were obtained from CLEA Japan, Inc. (Tokyo, Japan). The animals were maintained under pathogen-free conditions, in accordance with institutional principles. All animal experiments were approved by Kobe
University Animal Experimentation Regulations (permission no. P-130807). Nara-H cells were implanted into the dorsal, subcutaneous area of mice $(n=18)$ at a dose of $1.0 \times 10^{6}$ cells in $500 \mu \mathrm{l}$ PBS, as previously described (23) and mice were randomly divided into three groups: YM155 at $4 \mathrm{mg}(\mathrm{n}=6)$, YM155 at $2 \mathrm{mg}(\mathrm{n}=6)$ and control $(\mathrm{n}=6)$. Treatment commenced 2 weeks after cell implantation by intraperitoneal injection of YM155 (2 or $4 \mathrm{mg} / \mathrm{kg}$ ) or saline (as control), five times/week for 2 weeks. Tumor volume was calculated, as previously described, according to the formula $\mathrm{V}=\pi / 6 \times \mathrm{a}^{2} \mathrm{x} b$, where $\mathrm{a}$ and $\mathrm{b}$ represent the shorter and the longer dimensions of the tumor, respectively (23). At the end of the experiments, all tumors were excised and stored at $-80^{\circ} \mathrm{C}$. Survivin expression was assessed by qPCR, and apoptotic activity was evaluated by FACS and immunofluorescence staining.

Quantitative real-time PCR ( $q P C R$ ). We isolated total RNAs from tumor tissues, cells or implanted tumors using an RNeasy Mini kit, according to the manufacturer's protocol (Qiagen, Valencia, CA, USA), and first-strand cDNAs were synthesized using a High Capacity cDNA Transcription kit (Applied Biosystems, Foster City, CA, USA). qPCR was performed in a 20- $\mu 1$ reaction mixture using the Power SYBR Green Master Mix reagent (Applied Biosystems) on an ABI PRISM 7500 sequence detection system (Applied Biosystems). The cycling conditions were as follows: 1 cycle at $95^{\circ} \mathrm{C}$ for $10 \mathrm{~min}$ followed by 40 cycles at $95^{\circ} \mathrm{C}$ for $15 \mathrm{sec}$ and $60^{\circ} \mathrm{C}$ for $1 \mathrm{~min}$. Primers for human survivin [5'-CTT GGC CCA GTG TTT CTT CT-3' (upstream) and 5'-CCT CCC AAA GTG CTG GTA TT-3' (downstream)] and the internal control, human $\beta$-actin [5'-AGT CCT GTG GCA TCC ACG AAA-3' (upstream) and 5'-GTC ATA CTC CTG CTT GCT GA-3' (downstream)] were synthesized by Applied Biosystems. The values were normalized with $\beta$-actin, and relative expression was analyzed using the $\Delta \Delta \mathrm{Ct}$ method.

Immunoblot analysis. Lysates were extracted from cells or implanted tumors using a whole cell lysis buffer (Mammalian Protein Extraction reagent, Thermo Scientific, Rockford, IL, USA) supplemented with a protease and phosphatase inhibitor mix (Roche Applied Science, Indianapolis, IN, USA). Protein content was quantified using the BCA Protein Assay reagent (Bio-Rad, Hercules, CA, USA). Samples containing equal amounts of protein were electrophoresed through 7.5-15\% SDS-PAGE gradient gels and transferred onto PVDF membranes. After blocking, membranes were incubated overnight at $4^{\circ} \mathrm{C}$ with the following antibodies in CanGet Signal Solution 1 (Toyobo Co., Ltd., Osaka, Japan): anti-human survivin $(1: 1,000)$, anti-human PARP $(1: 1,000)$, anti-human cleaved PARP $(1: 1,000)$, anti-human caspase-3 $(1: 1,000)$, anti-human cleaved caspase-3 (1:500), anti-human caspase-9 (1:1,000), and anti-human cleaved caspase-9 (1:500). All antibodies were purchased from Cell Signaling Technology (Denvers, MA, USA). Following washes, membranes were incubated with the appropriate secondary antibody conjugated to horseradish peroxidase and were exposed with ECL Plus western blot detection system reagent (GE Healthcare Biosciences, Piscataway, NJ, USA). Protein expression was detected by Chemilumino analyzer LAS-3000 mini (Fujifilm, Tokyo, Japan). Membranes were reprobed with anti-human 
$\alpha$-tubulin antibody (Sigma-Aldrich) to confirm equal protein loading.

DNA fragmentation assays. DNA fragmentation was assessed by FACS analysis using the APO-DIRECT kit, according to the manufacturer's protocol (BD Pharmingen, Franklin Lakes, NJ, USA) (9). To obtain a single cell suspension from implanted tumor tissues, tumors were excised, minced, and filtered through a cell strainer (BD Falcon, Bedford, MA, USA). Erythrocytes were lysed in BD Pharm Lyse ${ }^{\mathrm{TM}}$ Lysing Buffer (BD Pharmingen) and the remaining cells were pelleted and resuspended in PBS. Single cell suspensions were fixed in $1 \%$ $(\mathrm{v} / \mathrm{v})$ paraformaldehyde and resuspended in $70 \%(\mathrm{v} / \mathrm{v})$ ice cold ethanol at a concentration of $1.0 \times 10^{6}$ cells $/ \mathrm{ml}$. Each cell pellet was resuspended in $50 \mu \mathrm{l}$ of DNA labeling solution (reaction buffer, $10 \mu \mathrm{l}$; TdT enzyme, $0.75 \mu \mathrm{l}$; FITC-dUTP, $8.0 \mu \mathrm{l}$; $\mathrm{dH}_{2} \mathrm{O}$, $31.25 \mu \mathrm{l}$ ) and incubated for $60 \mathrm{~min}$ at $37^{\circ} \mathrm{C}$. FITC-dUTPlabeled cells were analyzed.

Cell proliferation assays. We assessed cell proliferative activities in siRNA-transfected or YM155-treated cells by WST-8 assay using Cell Counting Kit-8 (CCK-8; Dojindo Inc., Kumamoto, Japan). Cells were seeded in 96-well culture plates at a density of $5 \times 10^{3}$ cells/well in $100 \mu \mathrm{l}$ culture medium. At the indicated times after siRNA transfection or YM155, $10 \mu \mathrm{l}$ of the CCK-8 solution was added, and optical density was measured at $450 \mathrm{~nm}$ using a Model 680 Microplate Reader (Bio-Rad). The relative number of viable cells in each well was calculated.

Immunofluorescence staining. Formalin-fixed, paraffinembedded tumor sections $(5 \mathrm{~mm})$ were pretreated with citrate buffer for $40 \mathrm{~min}$ at $95^{\circ} \mathrm{C}$, quenched with $0.05 \% \mathrm{H}_{2} \mathrm{O}_{2}$, and incubated with primary antibody overnight at $4^{\circ} \mathrm{C}$. Apoptotic activity was assessed in treated tumors by immunofluorescence staining using the APO-DIRECT kit, following the manufacturer's protocol (BD Pharmingen). The nuclei were stained with DAPI. The images were obtained using a BZ-8100 confocal microscope (Keyence, Osaka, Japan).

Statistical analysis. Each experiment was performed independently at least three times. ANOVA with post hoc test was used to compare for continuous values. All tests were considered significant at $\mathrm{p}<0.05$. Data were presented as the mean $\pm \mathrm{SE}$. For distributed data, the two-tailed Mann-Whitney U test was used for comparison between groups.

\section{Results}

Survivin expression is higher in MFH/UPS tissues. We first surveyed survivin mRNA expression in human muscloskeletal tumor tissue samples, and found that survivin mRNA expression in MFH/UPS samples was significantly higher than expression in samples from benign schwannomas. The relative expression of survivin increased 32-fold in MFH/UPS samples compared to that in benign schwannomas ( ${ }^{*} \mathrm{p}<0.05$, Fig. 1$)$.

siRNA knockdown of survivin affects mitochondrial apoptosis and cell proliferation. In qPCR, survivin-siRNA transfection significantly suppressed survivin mRNA expression in

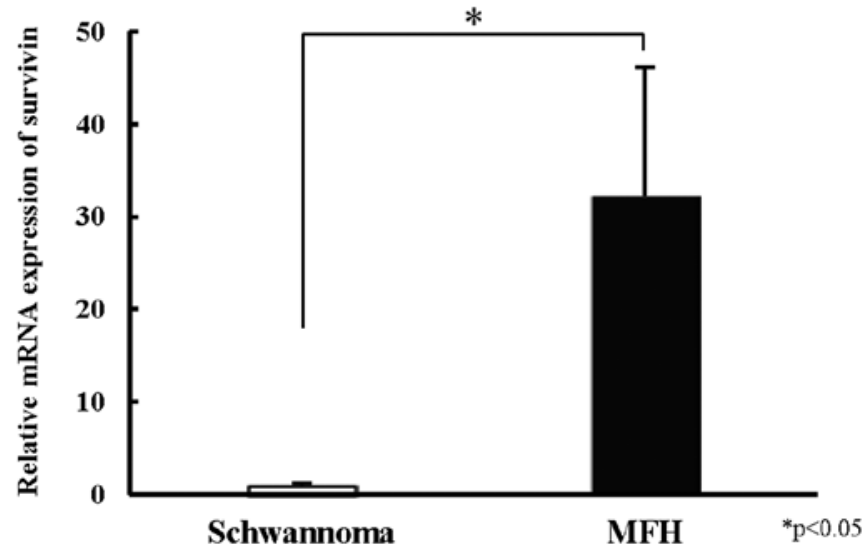

Figure 1. Evaluation of survivin mRNA expression in human musculoskeletal tumor tissue samples. The relative survivin mRNA expression was evaluated in 15 benign schwannomas and $15 \mathrm{MFH}$ /UPSs by qPCR. Results were normalized to the mean value of the schwannomas. Data represent mean $\pm \mathrm{SE}$ of at least three independent experiments $\left({ }^{*} \mathrm{p}<0.05\right)$.

human MFH/UPS cells compared to control siRNA ( ${ }^{*} \mathrm{p}<0.05$, Fig. 2A). Consistent with these results, immunoblot analyses showed that survivin protein expression decreased with survivin-siRNA transfection (Fig. 2B).

To evaluate the effects of survivin-siRNA on cellular apoptosis, we assessed DNA fragmentation and the expression of apoptosis-related proteins in siRNA-transfected Nara-H cells. Immunoblot analyses indicated that the expression of the cleaved forms of caspase-3, -9, and PARP increased in survivin-siRNA transfected cells $72 \mathrm{~h}$ post-transfection, while the expression of those proteins in control cells was weakly detected (Fig. 2C). In FACS analysis, DNA fragmentation increased in survivin-siRNA transfected cells in a time-dependent manner when compared to control cells (Fig. 2D). To identify the effect of survivin knockdown on MFH/UPS cell viability, we performed cell proliferation assays in siRNA-transfected cells. In all three MFH/UPS cell lines, cell viabilities were significantly decreased in a timedependent manner ( ${ }^{*} \mathrm{p}<0.05$, Fig. $\left.2 \mathrm{E}\right)$.

YM155 increases apoptotic activity in human MFH/UPS cells in vitro. YM155 (10 $\mathrm{nM})$ strongly suppressed mRNA ( ${ }^{*} \mathrm{p}<0.05$, Fig. 3A) and protein (Fig. 3B) expression of survivin in human MFH/UPS cell lines after $72 \mathrm{~h}$ of treatment. In immunoblot analyses, expression of the cleaved forms of caspase-3, -9, and PARP increased in YM155-treated Nara-H cells in a dosedependent manner, while expression of the full length forms of caspase-3 and caspase-9 decreased (Fig. 3C). Consistent with this, DNA fragmentation increased in all YM155-treated MFH/UPS cell lines in a time-dependent manner (Fig. 3D). Additionally, cell proliferation assays showed that YM155, at concentrations of $10 \mathrm{nM}$ or more, significantly decreased cell proliferation in a dose- and a time-dependent manner in all three MFH/UPS cell lines (Fig. 3E).

Antitumor effects of YM155 on human MFH/UPS xenografts in vivo. We evaluated the in vivo antitumor activity of YM155 using human MFH/UPS xenografts. The final tumor volumes in the YM155 at $2 \mathrm{mg}$-treated and YM155 at 4 mg-treated 
A

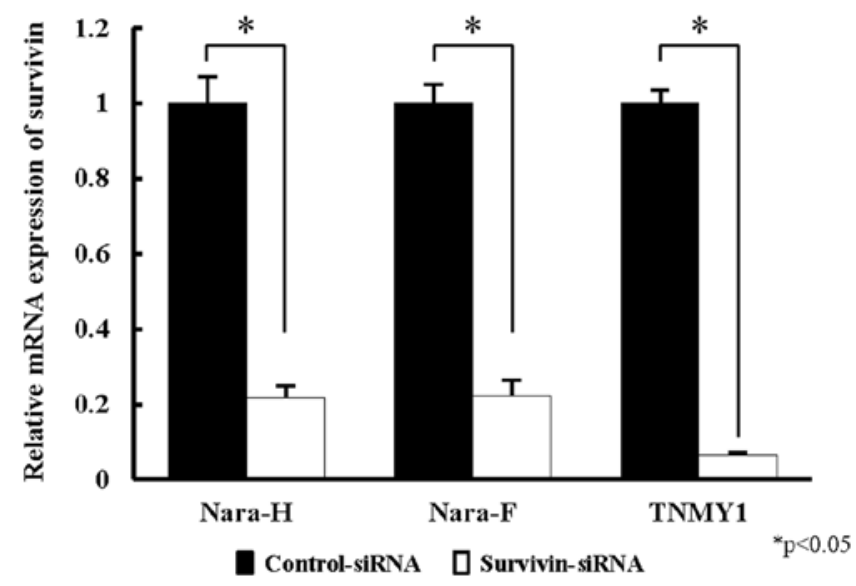

B
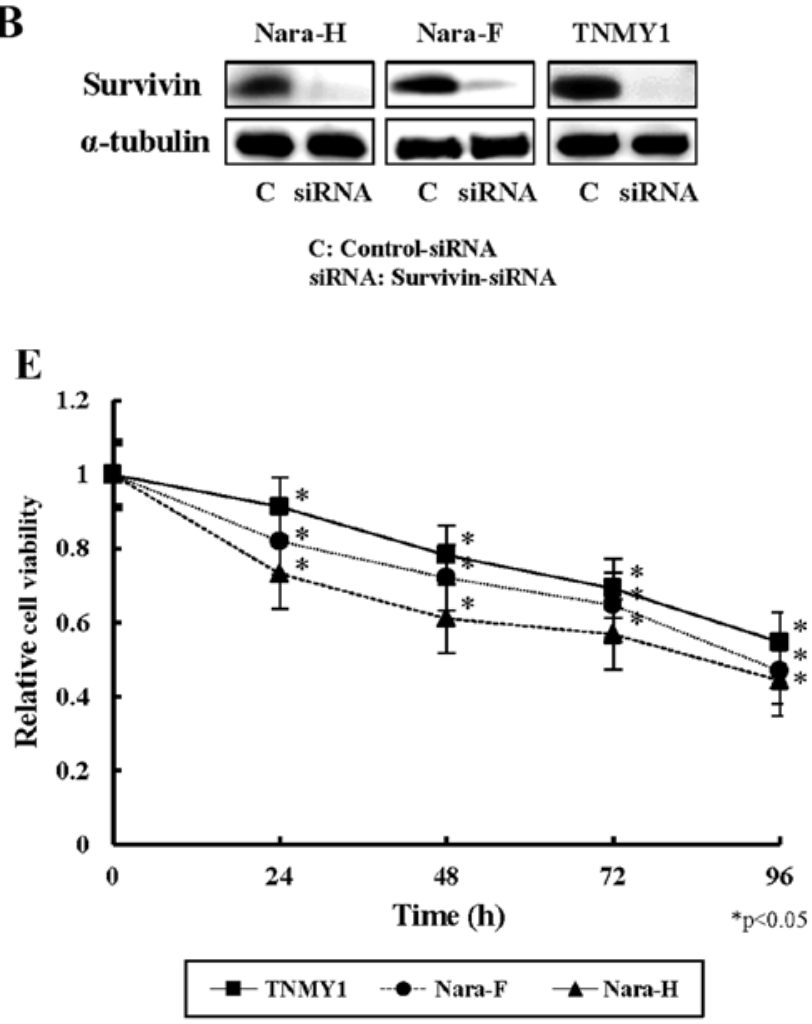

groups were 10.7 and $1.1 \%$, respectively, of that in the control group $(\mathrm{p}<0.05)$. No significant loss in body weight was observed during the experimental periods (Fig. 4A). Survivin mRNA (p<0.05, Fig. 4B) and protein (Fig. 4C) expression in YM155 treated tumors significantly decreased compared with control tumors. Using FACS analysis (Fig. 4D) and immunofluorescence staining (Fig. 4E), we found that apoptotic activity significantly increased after YM155 treatment, while survivin expression decreased.

\section{Discussion}

MFH/UPSs are clinically aggressive and highly metastatic $(1,2)$. Current therapeutic strategies for treating soft tissue sarcomas (STS) are ineffective, and patient prognosis is poor due to local recurrence and distant metastases (3). STSs are
C

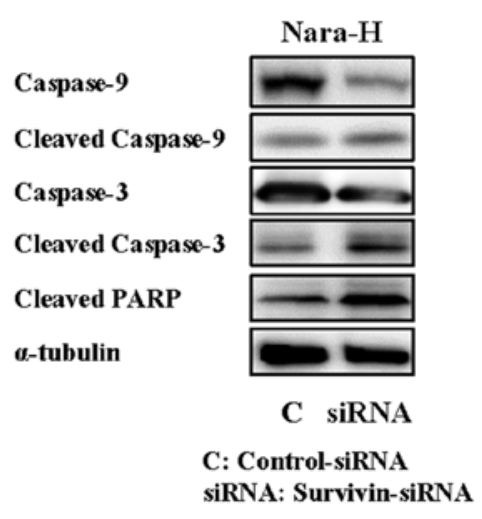

D

Nara-H

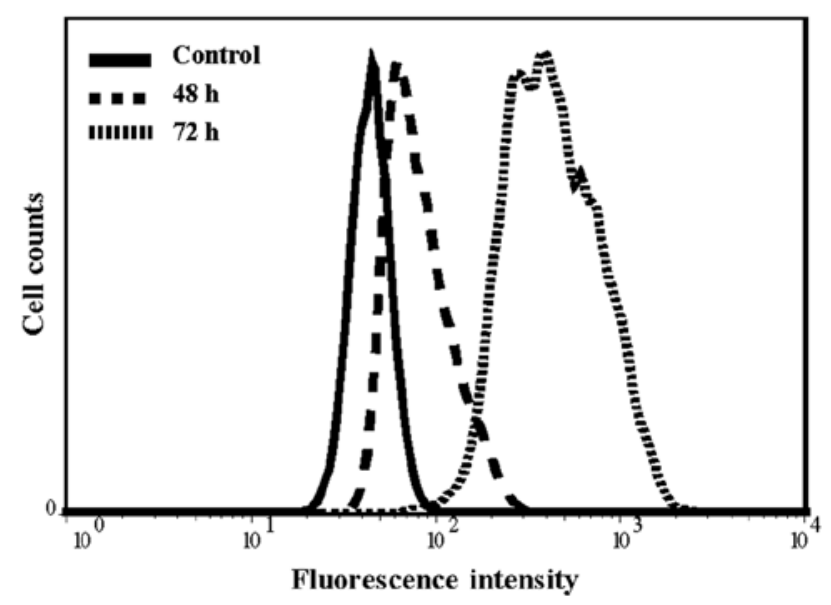

Figure 2. Effects of survivin siRNA on apoptotic activity and cell viability in human MFH/UPS cell lines. (A) Survivin mRNA expression was evaluated by qPCR in siRNA transfected MFH/UPS cell lines after $72 \mathrm{~h}$ of siRNA transfection. Data represent mean $\pm \mathrm{SE}$ of at least three independent experiments ( $(\mathrm{p}<0.05)$. (B) Immunoblot analysis for survivin in siRNA-transfected MFH/UPS cell lines. (C) Immunoblot analysis for apoptosis-related proteins in siRNA-transfected Nara-H MFH/UPS cells. (D) DNA fragmentation was assessed by FACS analysis in siRNA-transfected Nara-H MFH/UPS cells. (E) Relative cell viability was assessed by WST-8 assay in siRNA-transfected MFH/UPS cell lines after 24, 48, 72, and $96 \mathrm{~h}$ of siRNA transfection. Data represent mean $\pm \mathrm{SE}$ of at least three independent experiments $(\mathrm{p}<0.05)$.

histologically a broad group of rare tumors, accounting for $1 \%$ of all human malignant tumors. Radical surgery remains the primary mode of treatment for STSs; however, adjuvant chemotherapy or radiation therapy are desired early stage alternatives due to the high recurrence and metastatic rate of the tumor. Among the approved antitumor drugs, doxorubicin- and ifosfamide-based cytotoxic chemotherapies play an established role for STSs. Moreover, identification of the molecular pathways involved in STS tumorigenesis led to the development of molecular targeting agents which mainly inhibit tyrosine kinases, such as imatinib, used for advanced or metastatic dermatofibrosarcoma protuberans; pazopanib, approved as a second line regimen for advanced non-adipocitic STSs; and sunitinib, used for solitary fibrous tumor, alveolar soft part sarcoma, and extraskeletal myxoid chondrosarcoma $(24,25)$. Chemotherapeutic effects should be important in the 
A

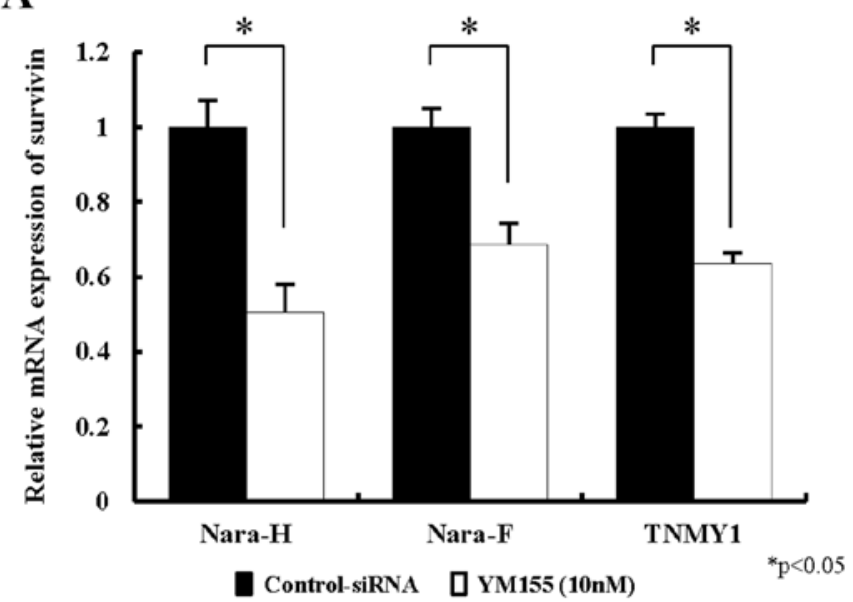

B

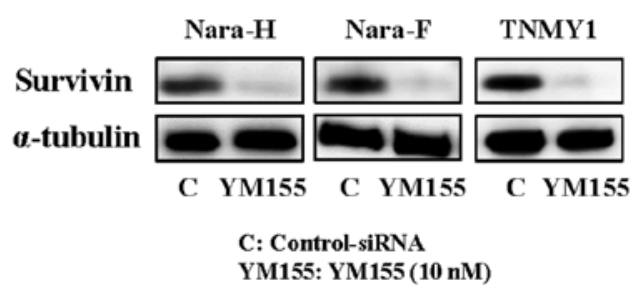

C

Caspase-9
Cleaved Caspase-9
Caspase-3
Cleaved Caspase-3
Cleaved PARP
a-tubulin

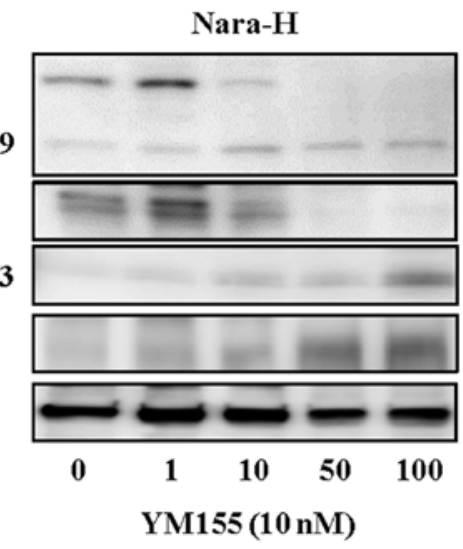

Figure 3. Effects of YM155 on apoptotic activity and cell viability in human MFH/UPS cell lines. (A) Survivin mRNA expression was evaluated by qPCR after $72 \mathrm{~h}$ of YM155 treatment $(10 \mathrm{nM})$. Data represent mean $\pm \mathrm{SE}$ of at least three independent experiments ( $\mathrm{p}<0.05)$. (B) Protein expression of survivin was assessed by immunoblot analysis in $10 \mathrm{nM}$ of YM155-treated human MFH/UPS cell lines. (C) Immunoblot analysis for apoptosis-related proteins in YM155-treated Nara-H cells. (D) DNA fragmentation after 48 and $72 \mathrm{~h}$ of YM155 treatment was assessed by FACS analysis in human MFH/UPS cell lines. (E) Relative cell viability was assessed by WST-8 assay in YM155 treated MFH/UPS cell lines after 24, 48, 72, and $96 \mathrm{~h}$ of treatment. Data represent mean $\pm \mathrm{SE}$ of at least three independent experiments $(\mathrm{p}<0.05)$.

D

Nara-H

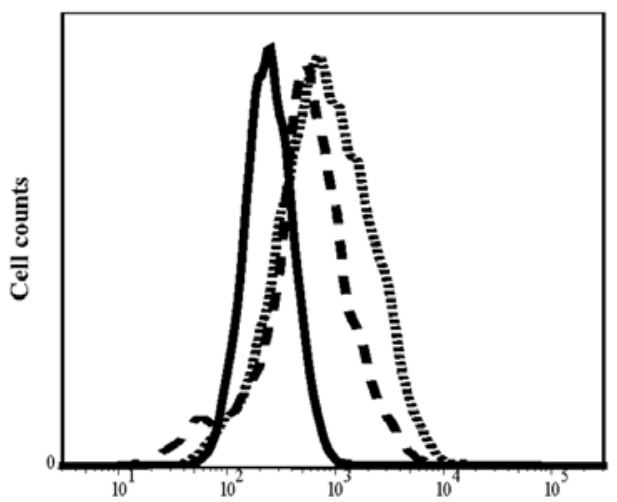

$\mathbf{E}$

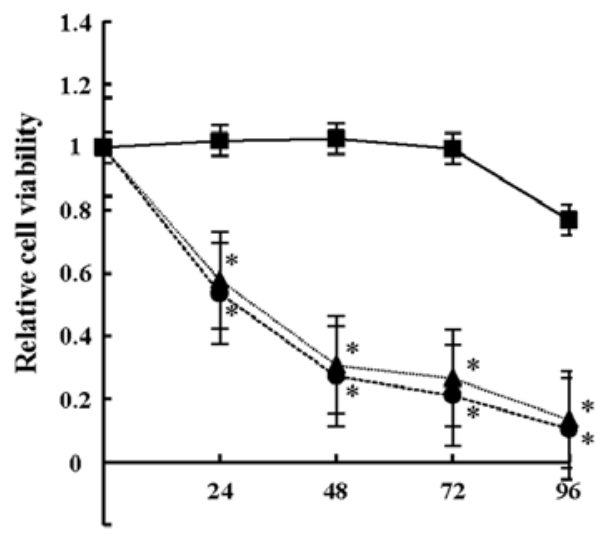

Nara-F

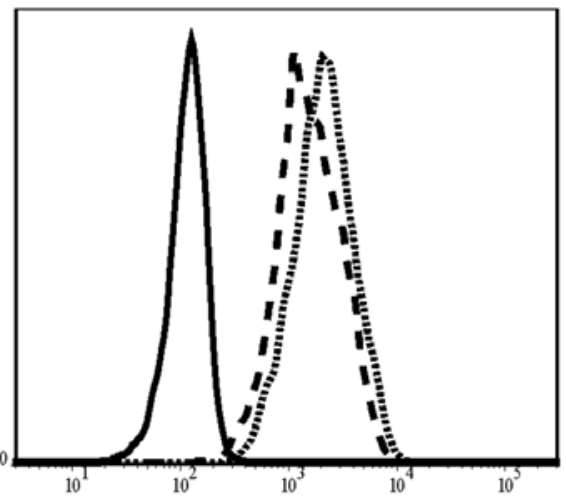

Fluorescence intensity

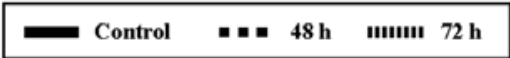

TNMY1

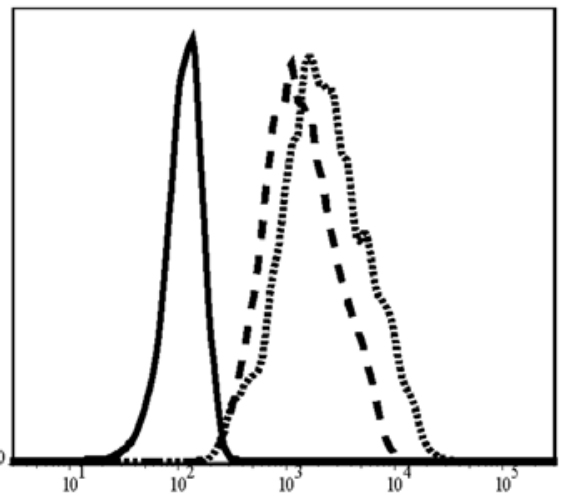

TNMY1
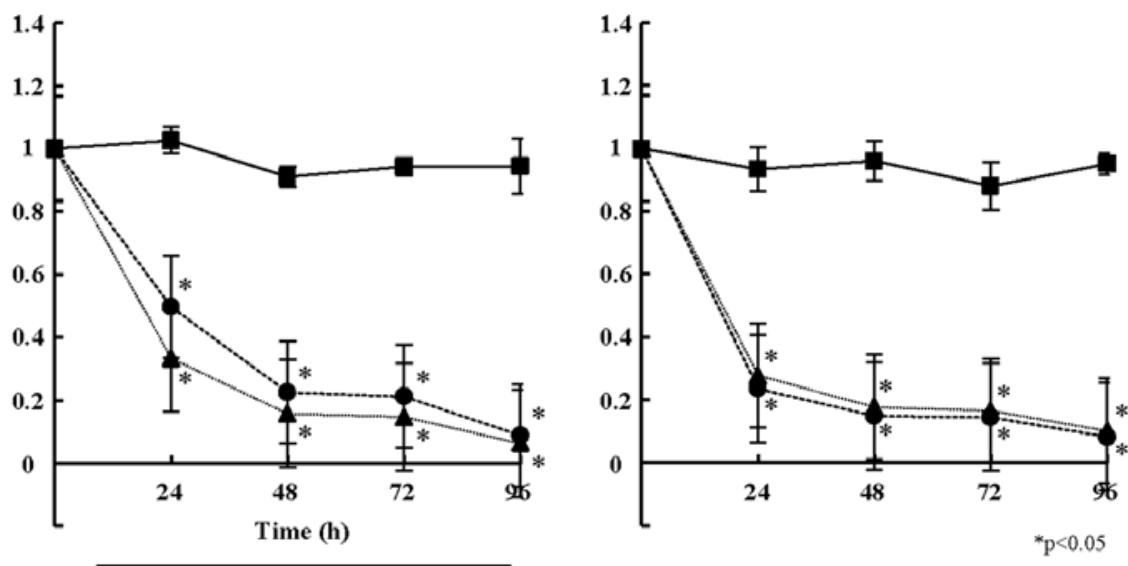

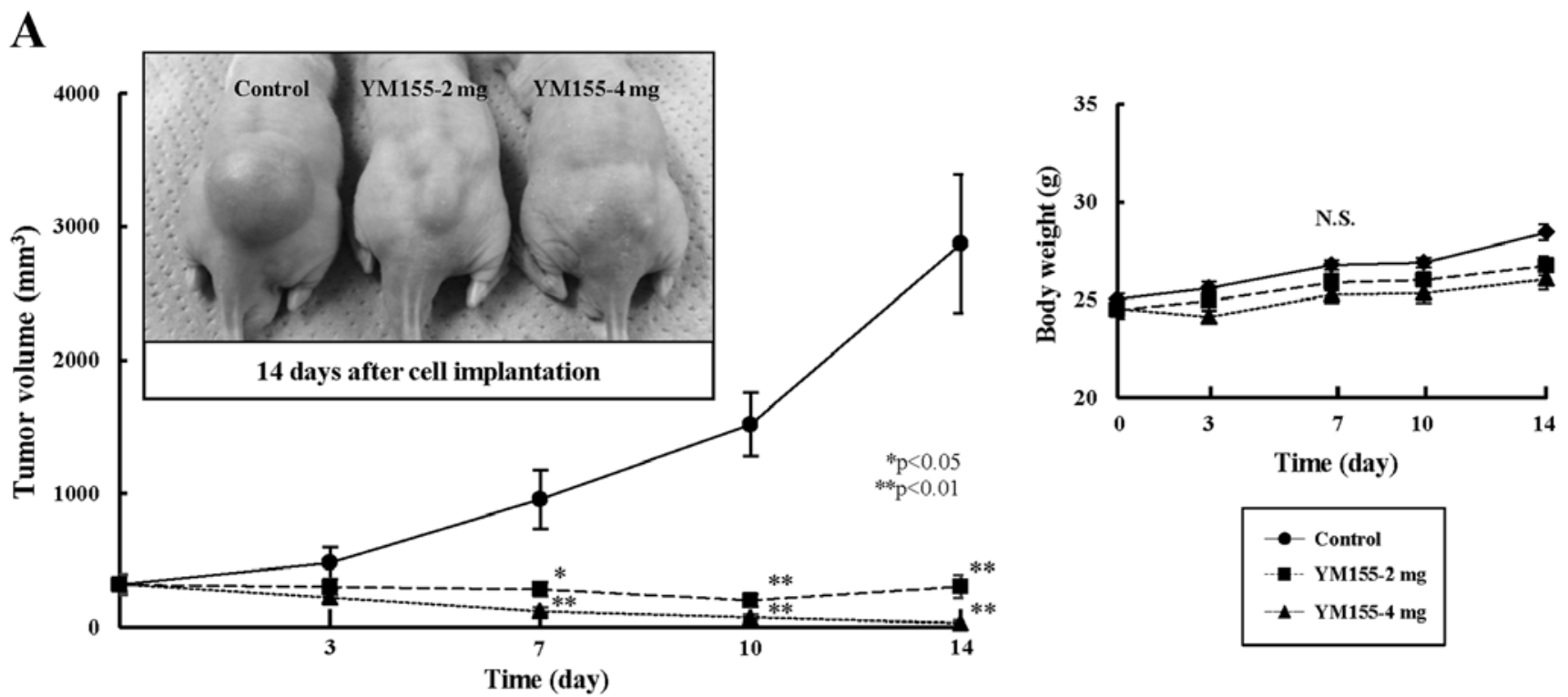

B

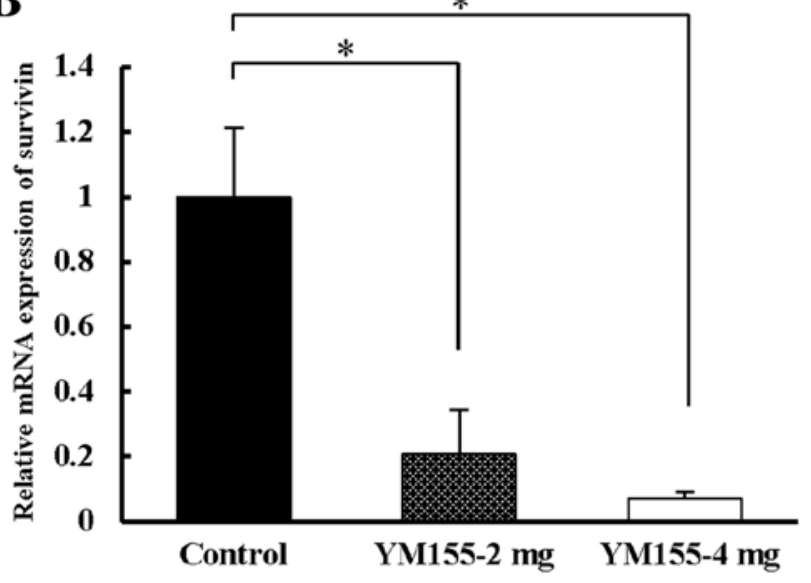

C

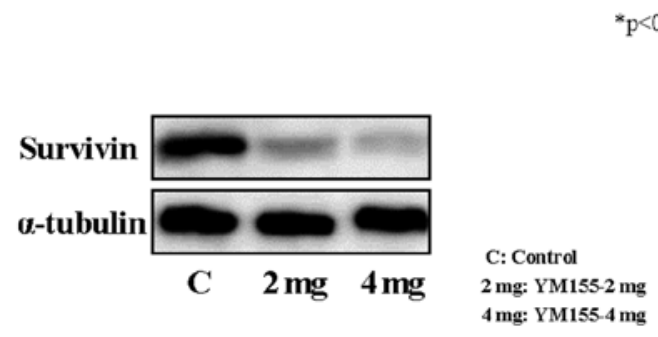

D

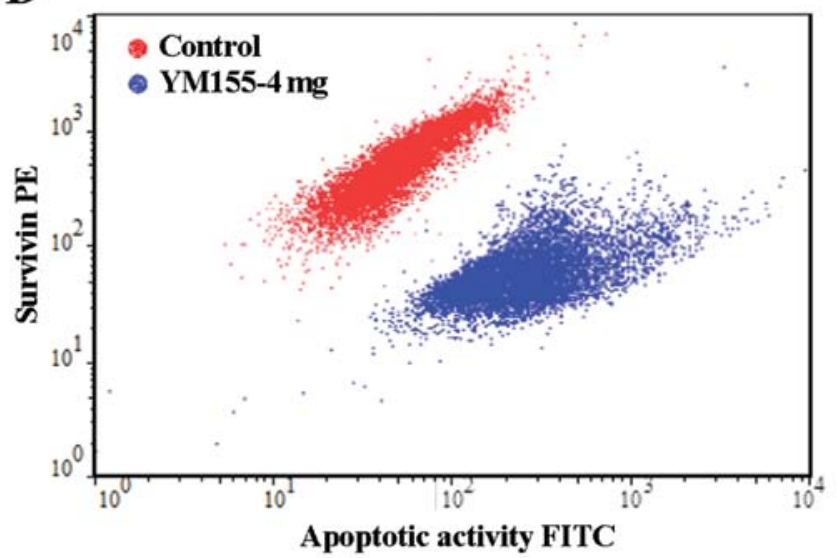

$\mathbf{E}$

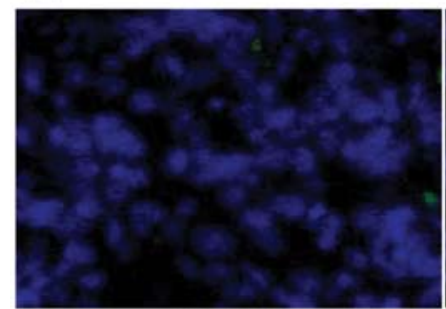

Control

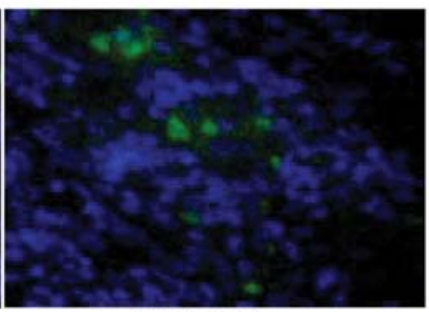

YM155-4mg
Blue: Nuclear (DAPI)

Green: Apoptotic nuclear (APO-DIRECT)

Figure 4. In vivo antitumor activities of YM155 on human MFH/UPS xenografts (Nara-H). (A) Tumor volume (mm³) and body weight (g) in YM155treated $(2 \mathrm{mg}$ or $4 \mathrm{mg})$ or control mice was monitored for 14 days. Data represent mean $\pm \mathrm{SE}$ of at least three independent experiments $\left(" \mathrm{p}<0.05,{ }^{* *} \mathrm{p}<0.01\right)$. (B) Survivin mRNA expression in YM155-treated or control tumors was evaluated by qPCR. Data represent mean \pm SE of at least three independent experiments ( $\mathrm{p}<0.05)$. (C) Immunoblot analysis for survivin in YM155-treated or control tumors. (D) Correlation between apoptotic activity and survivin expression in YM155-4 mg-treated or control tumors was assessed by FACS analysis (red, control; blue, YM155-4 mg). (E) DNA fragmentation analysis of tumor tissues from YM155-4 mg-treated and control mice by immunofluorescence staining [blue, nuclear (DAPI); green, apoptosis nuclear (APO-DIRECT)].

survival of patients with the disease, and this systemic modality should be the treatment of choice for patients at an advanced stage (26); however, the conventional chemotherapeutic strategies for high-grade STSs are ineffective and the prognosis of patients can be extremely poor because of local recurrence and metastases (3). Although studies have shown beneficial effects for tyrosine kinase inhibitors, new therapeutic strategies focusing on other molecular targets are required to be effective against high-grade STSs.

In this study, we examined the therapeutic potential of the inhibition of survivin, a member of the IAP family, on human MFH/UPS tumor growth. Survivin, the smallest 
member of the IAP family, is a 142-amino acid, 16.5-kDa protein coded by a single-copy gene on the human $17 q 25$ chromosome. Structurally, survivin contains a single repeat of the characteristic baculovirus IAP domain, essential for the caspase-inhibitory function, and an extended carboxy-terminal $\alpha$-helical coiled coil, but does not contain other identifiable domains (27-29). Resistance to apoptosis is a hallmark of malignant tumors; overexpression of IAP proteins enhances resistance to apoptotic stimuli in various malignancies (30). Among this protein family, survivin has taken a center stage because of its markedly specific expression in cancer cells $(8,28)$. In the present study, we surveyed survivin expression in human musculoskeletal tumor tissue samples, and found that survivin was highly expressed in human MFH/UPS. This result suggests that elevated survivin expression strongly influences tumor progression in malignant musculoskeletal tumors.

Survivin is a bi-functional IAP that has been implicated in protection from apoptosis and regulation of mitosis (31). Initially, survivin was described as an inhibitor of caspase-9 (32); however, several studies have shown that the role of survivin in cancer pathogenesis is not limited to the inhibition of apoptosis but also involves regulation of the mitotic spindle checkpoint, and promotion of angiogenesis and chemoresistance (28,33-36). Although many functions for survivin have been identified, the mechanism by which survivin inhibits apoptosis has remained elusive. In particular, it is not clear whether survivin has a genuine function in the inhibition of apoptosis that is independent of its role as a master regulator of mitosis, and whether this function is important in disease pathogenesis. Recent experiments show that survivin inhibits active caspase- 9 but not active caspase- 3 and -7 , and that this inhibition requires the IAP family member, X-linked IAP (XIAP) (35), and the hepatitis B X-interacting protein (37).

While undetectable in most adult differentiated tissues, survivin is ubiquitously expressed during embryonic development and is highly re-expressed in malignant tumors, including lung, breast, colorectal, gastric, prostate, hepatocellular, and renal cancers, as well as melanoma and STS (13,15,38-40). Studies suggest an interrelation between survivin expression and either poor disease prognosis or unresponsiveness to chemotherapy or radiation therapy $(39,41,42)$. These findings strongly suggest that suppressing survivin may substantially contribute to antitumor activity in cancer cells. Tsuji et al reported that dsRNA-mediated silencing of survivin noticeably reduces survivin mRNA and protein expression in the pancreatic cancer cell line PANC-1, thereby resulting in cell apoptosis while failing to influence the cell cycle (43). Wang et al showed that conjugate-mediated survivin-siRNA can efficiently target glioma tumors in vitro and in vivo, and that silencing survivin by this method prolonged the survival times of orthotopic tumor-bearing mice (44). In musculoskeletal malignancies, the survivin inhibition by survivin-specific siRNAs enhanced sensitivity to doxorubicin and cisplatin in a survivin-overexpressing osteosarcoma cell line (45). In the present study, we found that survivin-siRNA strongly suppressed survivin expression, and that survivin-siRNA significantly increased the number of apoptotic cells and decreased cell proliferation in human MFH/UPS cell lines. Therefore, survivin inhibition likely induces cell apoptosis, resulting in an antitumor effect on human MFH/UPS.
YM155, a novel small-molecule suppressor of survivin, suppresses survivin expression with little effect on the expression levels of other IAP family members. The exact molecular mechanism by which YM155 downregulates survivin expression at the mRNA level and, subsequently, at the protein level, is not known. A previous study indicated that YM155 treatment inhibits the growth of wide variety of cancer cell lines in vitro and demonstrates significant antitumor activity without causing body weight loss in xenograft models (4). The study indicated that tumor regression induced by YM155 was associated with reduced intratumoral survivin expression levels, increased apoptosis, and decreased mitotic index (4). In the present study, we demonstrated that YM155 treatment induced mitochondrial apoptosis and decreased cell proliferation in human MFH/UPS cells in vitro, and that YM155 suppressed in vivo MFH/UPS cell growth by inducing apoptosis without apparent body weight loss. Our results strongly suggest that survivin expression contributes to human MFH/ UPS tumor progression, and that survivin inhibition by either specific siRNA or by YM155, a novel survivin suppressant, provides an antitumor effect on human MFH/UPS via induction of apoptosis.

Considering that apoptosis is the primary mode of cell death induced by several classes of anticancer agents, a possible general role of survivin in determining the chemo-sensitivity profiles of tumor cells has been investigated and concluded that the suppression of survivin expression achieved increased sensitivity of various anticancer agents $(39,46,47)$. Faversani et al reported that high-throughput pharmacologic targeting of survivin family proteins with YM155 selectively potentiated the effect of doxorubicin, and induced tumor cell apoptosis in breast cancer cell types. This result suggests that incorporation of YM155 in anthracycline-containing chemotherapy may result in greater clinical activity across breast cancer subtypes, and potentially overcome constitutive treatment resistance (48). Clinically, there are several ongoing studies using YM155 $(18,19)$. Tolcher et al reported in a phase II study of YM155 that $25 \%$ of patients with castration-resistant prostate cancer had prolonged stable disease and that the tolerability regimen induced responses in the phase I trial. Survivin-targeting therapies can potentially activate the apoptotic pathways and induce mitochondrial activation (49). The first phase I/II study of YM155 with paclitaxel and carboplatin in patients with advanced non-small cell lung cancer (NSCLC) indicated that the administration of YM155 in combination with carboplatin and paclitaxel every 3 weeks resulted in a favorable safety profile but did not appear to significantly improve the response rate in advanced NSCLC. The authors concluded that combinations targeting several components within apoptotic pathways may be more effective (18). In the present study, we have proved that survivin inhibition shows antitumor effect on human MFH/UPS cells. Therefore, further in vivo studies should be conducted to test the efficacy of YM155 in the treatment of STS when used in combination therapy and we have no doubt that we can get better results particularly by the combination with doxorubicin.

In conclusion, our findings strongly indicate that survivin suppression induces mitochondrial apoptosis in human MFH/UPS cells, and that YM155 exhibits antiproliferative activity and induces tumor regression in human MFH/UPS 
xenografts. We propose that survivin suppressants, including YM155, be considered as potent therapeutic agents for the novel treatment of human MFH/UPS.

\section{Acknowledgements}

We thank Minako Nagata, Maya Yasuda, and Kyoko Tanaka for their expert technical assistance.

\section{References}

1. Spira AI and Ettinger DS: The use of chemotherapy in soft-tissue sarcomas. Oncologist 7: 348-359, 2002.

2. Matushansky I, Charytonowicz E, Mills J, Siddiqi S, Hricik T and Cordon-Cardo C: MFH classification: Differentiating undifferentiated pleomorphic sarcoma in the 21st Century. Expert Rev Anticancer Ther 9: 1135-1144, 2009.

3. Le Doussal V, Coindre JM, Leroux A, Hacene K, Terrier P, Bui NB, Bonichon F, Collin F, Mandard AM and Contesso G: Prognostic factors for patients with localized primary malignant fibrous histiocytoma: A multicenter study of 216 patients with multivariate analysis. Cancer 77: 1823-1830, 1996

4. Nakahara T, Kita A, Yamanaka K, Mori M, Amino N, Takeuchi M, Tominaga F, Kinoyama I, Matsuhisa A, Kudou M, et al: Broad spectrum and potent antitumor activities of YM155, a novel small-molecule survivin suppressant, in a wide variety of human cancer cell lines and xenograft models. Cancer Sci 102: 614-621, 2011.

5. Ren YQ, Zhang HY, Su T, Wang XH and Zhang L: Clinical significance of serum survivin in patients with pancreatic ductal adenocarcinoma. Eur Rev Med Pharmacol Sci 18: 3063-3068, 2014.

6. Caldas H, Holloway MP, Hall BM, Qualman SJ and Altura RA: Survivin-directed RNA interference cocktail is a potent suppressor of tumour growth in vivo. J Med Genet 43: 119-128, 2006.

7. Vischioni B, van der Valk P, Span SW, Kruyt FA, Rodriguez JA and Giaccone G: Nuclear localization of survivin is a positive prognostic factor for survival in advanced non-small-cell lung cancer. Ann Oncol 15: 1654-1660, 2004.

8. Mobahat M, Narendran A and Riabowol K: Survivin as a preferential target for cancer therapy. Int J Mol Sci 15: 2494-2516, 2014.

9. Altieri DC: Survivin, cancer networks and pathway-directed drug discovery. Nat Rev Cancer 8: 61-70, 2008.

10. Pennati M,Folini M and Zaffaroni N: Targeting survivin in cancer therapy: Fulfilled promises and open questions. Carcinogenesis 28: $1133-1139,2007$.

11. Span PN, Sweep FC, Wiegerinck ET, Tjan-Heijnen VC, Manders P, Beex LV and de Kok JB: Survivin is an independent prognostic marker for risk stratification of breast cancer patients. Clin Chem 50: 1986-1993, 2004.

12. Trieb K, Lehner R, Stulnig T, Sulzbacher I and Shroyer KR: Survivin expression in human osteosarcoma is a marker for survival. Eur J Surg Oncol 29: 379-382, 2003.

13. Wu YF, Liang XJ, Liu YY, Gong W, Liu JX, Wang XP, Zhuang ZQ, Guo $Y$ and Shen HY: +Antisense oligonucleotide targeting survivin inhibits growth by inducing apoptosis in human osteosarcoma cells MG-63. Neoplasma 57: 501-506, 2010.

14. Liang X, Da M, Zhuang Z, Wu W, Wu Z, Wu Y and Shen H: Effects of Survivin on cell proliferation and apoptosis in MG-63 cells in vitro. Cell Biol Int 33: 119-124, 2009.

15. Lechler P, Renkawitz T, Campean V, Balakrishnan S, Tingart M, Grifka J and Schaumburger J: The antiapoptotic gene survivin is highly expressed in human chondrosarcoma and promotes drug resistance in chondrosarcoma cells in vitro. BMC Cancer 11: $120,2011$.

16. Taubert H, Kappler M, Bache M, Bartel F, Köhler T, Lautenschläger C, Blümke K, Würl P, Schmidt H, Meye A, et al: Elevated expression of survivin-splice variants predicts a poor outcome for soft-tissue sarcomas patients. Oncogene 24 $5258-5261,2005$

17. Nakahara T,Kita A, Yamanaka K, Mori M, Amino N, Takeuchi M, Tominaga F, Hatakeyama S, Kinoyama I, Matsuhisa A, et al: YM155, a novel small-molecule survivin suppressant, induces regression of established human hormone-refractory prostate tumor xenografts. Cancer Res 67: 8014-8021, 2007.
18. Kelly RJ, Thomas A, Rajan A, Chun G,Lopez-Chavez A, Szabo E, Spencer S, Carter CA, Guha U, Khozin S, et al: A phase I/II study of sepantronium bromide (YM155, survivin suppressor) with paclitaxel and carboplatin in patients with advanced non-smallcell lung cancer. Ann Oncol 24: 2601-2606, 2013.

19. Satoh T, Okamoto I, Miyazaki M, Morinaga R, Tsuya A, Hasegawa Y, Terashima M, Ueda S, Fukuoka M, Ariyoshi Y, et al: Phase I study of YM155, a novel survivin suppressant, in patients with advanced solid tumors. Clin Cancer Res 15: 3872-3880, 2009.

20. Cheson BD, Bartlett NL, Vose JM, Lopez-Hernandez A, Seiz AL, Keating AT, Shamsili S and Papadopoulos KP: A phase II study of the survivin suppressant YM155 in patients with refractory diffuse large B-cell lymphoma. Cancer 118: 3128-3134, 2012.

21. Kiyozuka Y, Nakagawa H, Uemura Y, Senzaki H, Yamamoto A, Noguchi T, Mizuta H, Nakanishi K, Nakano S and Tsubura A: Novel cell lines established from a human myxoid malignant fibrous histiocytoma arising in the uterus. Cancer Genet Cytogenet 127: 7-15, 2001.

22. Nakatani T, Marui T, Yamamoto T, Kurosaka M, Akisue T and Matsumoto K: Establishment and characterization of cell line TNMY1 derived from human malignant fibrous histiocytoma. Pathol Int 51: 595-602, 2001.

23. Okada Y, Akisue T, Hara H, Kishimoto K, Kawamoto T, Imabori M, Kishimoto S, Fukase N, Onishi Y and Kurosaka M: The effect of bevacizumab on tumour growth of malignant fibrous histiocytoma in an animal model. Anticancer Res 30: 3391-3395, 2010.

24. Radaelli S, Stacchiotti S, Casali PG and Gronchi A: Emerging therapies for adult soft tissue sarcoma. Expert Rev Anticancer Ther 14: 689-704, 2014.

25. Ranieri G, Mammì M, Donato Di Paola E, Russo E, Gallelli L, Citraro R, Gadaleta CD, Marech I, Ammendola M and De Sarro G: Pazopanib a tyrosine kinase inhibitor with strong anti-angiogenetic activity: A new treatment for metastatic soft tissue sarcoma. Crit Rev Oncol Hematol 89: 322-329, 2014.

26. Santoro A, Tursz T, Mouridsen H, Verweij J, Steward W, Somers R, Buesa J, Casali P, Spooner D, Rankin E, et al: Doxorubicin versus CYVADIC versus doxorubicin plus ifosfamide in first-line treatment of advanced soft tissue sarcomas: A randomized study of the European Organization for Research and Treatment of Cancer Soft Tissue and Bone Sarcoma Group. J Clin Oncol 13: 1537-1545, 1995.

27. Sanna MG, da Silva Correia J, Ducrey O, Lee J, Nomoto K, Schrantz N, Deveraux QL and Ulevitch RJ: IAP suppression of apoptosis involves distinct mechanisms: The TAK1/JNK1 signaling cascade and caspase inhibition. Mol Cell Biol 22: 1754-1766, 2002

28. Mita AC, Mita MM, Nawrocki ST and Giles FJ: Survivin: Key regulator of mitosis and apoptosis and novel target for cancer therapeutics. Clin Cancer Res 14: 5000-5005, 2008.

29. Ambrosini G, Adida C and Altieri DC: A novel anti-apoptosis gene, survivin, expressed in cancer and lymphoma. Nat Med 3: 917-921, 1997.

30. Nachmias B, Ashhab Y and Ben-Yehuda D: The inhibitor of apoptosis protein family (IAPs): An emerging therapeutic target in cancer. Semin Cancer Biol 14: 231-243, 2004.

31. Altieri DC: Validating survivin as a cancer therapeutic target. Nat Rev Cancer 3: 46-54, 2003.

32. Altieri DC: Survivin, versatile modulation of cell division and apoptosis in cancer. Oncogene 22: 8581-8589, 2003.

33. Adida C, Berrebi D, Peuchmaur M, Reyes-Mugica M and Altieri DC: Anti-apoptosis gene, survivin, and prognosis of neuroblastoma. Lancet 351: 882-883, 1998.

34. Lin CY, Hung HC, Kuo RC, Chiang CP and Kuo MY: Survivin expression predicts poorer prognosis in patients with areca quid chewing-related oral squamous cell carcinoma in Taiwan. Oral Oncol 41: 645-654, 2005.

35. Reed JC: The Survivin saga goes in vivo. J Clin Invest 108: 965-969, 2001

36. Dohi T, Okada K, Xia F, Wilford CE, Samuel T, Welsh K, Marusawa $\mathrm{H}$, Zou H, Armstrong R, Matsuzawa S, et al: An IAP-IAP complex inhibits apoptosis. J Biol Chem 279: 34087-34090, 2004

37. Marusawa H, Matsuzawa S, Welsh K, Zou H, Armstrong R, Tamm I and Reed JC: HBXIP functions as a cofactor of survivin in apoptosis suppression. EMBO J 22: 2729-2740, 2003.

38. Fukuda S and Pelus LM: Survivin, a cancer target with an emerging role in normal adult tissues. Mol Cancer Ther 5: $1087-1098,2006$ 
39. Zaffaroni N, Pennati M, Colella G, Perego P, Supino R, Gatti L, Pilotti S, Zunino F and Daidone MG: Expression of the antiapoptotic gene survivin correlates with taxol resistance in human ovarian cancer. Cell Mol Life Sci 59: 1406-1412, 2002.

40. Granziero L, Ghia P, Circosta P, Gottardi D, Strola G, Geuna M, Montagna L, Piccoli P, Chilosi M and Caligaris-Cappio F: Survivin is expressed on CD40 stimulation and interfaces proliferation and apoptosis in B-cell chronic lymphocytic leukemia. Blood 97: 2777-2783, 2001.

41. Rödel F, Hoffmann J, Distel L, Herrmann M, Noisternig T, Papadopoulos T, Sauer R and Rödel C: Survivin as a radioresistance factor, and prognostic and therapeutic target for radiotherapy in rectal cancer. Cancer Res 65: 4881-4887, 2005.

42. Kappler M, Köhler T, Kampf C, Diestelkötter P, Würl P, Schmitz M, Bartel F, Lautenschläger C, Rieber EP, Schmidt H, et al: Increased survivin transcript levels: An independent negative predictor of survival in soft tissue sarcoma patients. Int J Cancer 95: 360-363, 2001.

43. Tsuji N, Asanuma K, Kobayashi D, Yagihashi A and Watanabe N: Introduction of a survivin gene-specific small inhibitory RNA inhibits growth of pancreatic cancer cells. Anticancer Res 25B: 3967-3972, 2005.

44. Wang F, Bai HR, Wang J, Bai YZ and Dou CW: Glioma growth inhibition in vitro and in vivo by single chain variable fragments of the transferrin receptor conjugated to survivin small interfering RNA. J Int Med Res 39: 1701-1712, 2011.
45. Wang J-W, Liu Y, Tian $\mathrm{H}$ and Zhang W: Effect of survivinsiRNA on drug sensitivity of osteosarcoma cell line MG-63. Chin J Cancer Res 22: 68-72, 2010.

46. Zaffaroni N, Pennati M and Daidone MG: Survivin as a target for new anticancer interventions. J Cell Mol Med 9: 360-372, 2005.

47. Ling X, Bernacki RJ, Brattain mg and Li F: Induction of survivin expression by taxol (paclitaxel) is an early event, which is independent of taxol-mediated G2/M arrest. J Biol Chem 279: 15196-15203, 2004.

48. Faversani A, Vaira V, Moro GP, Tosi D, Lopergolo A, Schultz DC, Rivadeneira D, Altieri DC and Bosari S: Survivin family proteins as novel molecular determinants of doxorubicin resistance in organotypic human breast tumors. Breast Cancer Res 16: R55, 2014.

49. Tolcher AW, Quinn DI, Ferrari A, Ahmann F, Giaccone G, Drake T, Keating A and de Bono JS: A phase II study of YM155, a novel small-molecule suppressor of survivin, in castrationresistant taxane-pretreated prostate cancer. Ann Oncol 23: 968-973, 2012 